\title{
Enquête
}

Archives de la revue Enquête

5 | 1989

Biographie et cycle de vie

\section{Cheminement régressif et expression héroïque du moi dans le récit de vie}

\section{Martine Burgos}

\section{(2) OpenEdition}

Journals

Édition électronique

URL : http://journals.openedition.org/enquete/108

DOI : 10.4000/enquete. 108

ISSN : 1953-809X

Éditeur :

Cercom, Éditions Parenthèses

Édition imprimée

Date de publication : 2 mars 1989

\section{Référence électronique}

Martine Burgos, "Cheminement régressif et expression héroïque du moi dans le récit de vie », Enquête [En ligne], 5 | 1989, mis en ligne le 27 juin 2013, consulté le 01 mai 2019. URL : http:// journals.openedition.org/enquete/108; DOI : 10.4000/enquete.108 


\title{
Cheminement régressif et expression héroïque du moi dans le récit de vie
}

\author{
Martine Burgos
}

1 La question que j'ai l'intention de traiter avec vous est celle de la périodisation dans les récits de vie. En analysant quelques récits de vie collectés auprès de femmes de la campagne et d'habitantes d'un quartier du centre de Paris, je me suis demandée s'il était possible de faire ressortir une quelconque similarité entre les différents événements narrés qui figurent comme étapes de transition ou de rupture, inaugurant une période nouvelle dans l'existence des narrateurs. Autrement dit, pouvons-nous dresser une sorte de typologie des événements repérables comme marqueurs de rupture dans la continuité biographique, ce qui permettrait d'établir un certain niveau de comparabilité entre les récits ? Si tel est le cas, se pose alors la question de la nature des événements en question : sont-ils d'ordre strictement personnel, privé, ou bien se rapportent-ils plutôt à un vécu collectif, celui que la mémoire historique formule et transmet par l'entremise des différentes institutions qui ont en charge sa gestion, l'institution scolaire en particulier?

2 Une fois qu'on a répondu à cette question, un autre problème se pose : en tant que récit, le récit de vie possède une logique interne qui est celle de l'élaboration d'un sens, quand bien même celui-ci se résumerait à la reconnaissance de l'absurdité, ou de la faillite, de toute quête de sens dans la vie, reconnaissance qui, renouant avec la tradition romantique de l'opposition de l'idéal et de la vie, ouvre le champ à la quête esthétique du sens. Il faudra donc se demander comment s'établit la relation entre ces deux séries d'événements, personnels et collectifs, que tisse le récit pour nous en donner une représentation unifiée par la recherche d'une cohérence. Je fais l'hypothèse que cette cohérence renvoie à la capacité d'un sujet à surmonter les événements historiques en les transformant en épreuves, moments cruciaux de sa quête personnelle, et, inversement, à donner à certaines actions, certains faits biographiques singuliers, la dimension d'un geste, au sens épique du terme. De fait, cette capacité n'est pas du seul ressort d'un individu exceptionnellement doué pour ce genre d'opération: elle lui est enseignée par 
les modèles narratifs que véhicule le discours social qui, à un moment donné, articule d'une certaine façon l'expression des expériences vécues et des projets individuels au champ socialement déterminé des possibles. Ainsi, certains types d'organisation sociale, celles que Louis Dumont désigne par le terme de sociétés "holistes", autorisent l'expression de la relation de l'individu à la communauté sous forme de récit épique, d'autres, renversant la hiérarchie des valeurs, privilégiant les aspirations de l'individu par rapport aux exigences de la société globale qui n'est plus perçue comme une réalité trans-individuelle, transcendantale, mais comme simple contexte, environnement social, vont autoriser l'émergence de la forme narrative moderne par excellence : le roman. Ce sont des exemples suffisamment connus et je ne m'y attarderai pas.

3 De fait, le récit, indépendamment de son contenu d'idées, qui peut résolument viser la subversion de l'ordre actuel, ne remet cependant jamais en question la nécessité d'un ordre, qu'il le pose comme passé perpétué dans le présent ou idéal passé définitivement perdu, futur où la nature humaine serait idéalement réconciliée avec elle-même, ou incontournable réalité qui permet à l'individu de se poser en s'opposant, selon la célèbre formule. La place d'un individu à l'intérieur du système social, concrétisée par le type de relation qu'il entretient avec les diverses médiations institutionnelles qui permettent à l'individu de se définir par rapport à la totalité du social (famille, insertion professionnelle, habitat, etc.) le prédispose à emprunter telle ou telle forme narrative parmi les quatre modèles précités. Il est probable que dans les sociétés individualistes modernes, les formes les plus courantes d'expression de soi emprunteront plus volontiers les traits du romanesque que ceux de l'épopée - ce que nous avons pu vérifier empiriquement par l'analyse des récits de vie d'anciennes agricultrices de la Sarthe, prises entre deux modèles narratifs, l'ancien (épique) et le moderne (romanesque), entre lesquels seule l'expérience concrète de l'exclusion hors de la communauté originelle offre une possibilité de choix, c'est-à-dire d'expression du procès d'individuation qui caractérise l'expérience moderne du sujet.

4 Quelle que soit l'importance du rôle qu'on attribue, dans la sélection et la mise en forme des contenus du récit de vie, aux structures narratives préexistant au projet même de raconter sa vie, il est cependant indéniable que le récit de vie, contrairement au pur récit de fiction, produit ce que Kate Hamburger appelle un énoncé de réalité à l'intérieur d'un genre mixte, dont relève l'autobiographie, qui combine fictionnalité et référentialité : en effet, si le récit de vie reconstruit un passé, le (dé)(re)(ré)forme - on a un vaste choix de termes -, ce qu'il est important de souligner, c'est que le récit de vie ne saurait jamais rompre complètement avec le réel, sauf à remettre en question le pacte implicite qui lie le narrateur avec son auditoire. Malgré toutes les déformations inhérentes à la posture rétrospective - décelables lorsque la mémoire s'exerce sur la réalité la plus factuelle d'une existence, dates et lieux, car en ce qui concerne la réalité "subjective», les jugements de valeurs, celle-ci échappe naturellement à tout contrôle empirique - le récit de vie prétend bien dire la vérité du sujet sur quelque chose qui reste relativement extérieur au procès d'énonciation puisqu'il est supposé, à travers l'histoire du narrateur, reproduire la genèse du sujet d'énonciation au prix d'une objectivation, d'une distanciation de ce dernier avec le (les) Moi, sujet(s) de l'énoncé ; simultanément, le sujet d'énonciation, s'imposant comme narrateur, élabore la figuration de son unité à travers la cohérence d'un récit qui subordonne la multiplicité des moments passés au présent de la narration; il construit ainsi son moi - son fantasme de moi, son moi fictionnel - son identité, à partir d'un récit dont la nécessaire cohérence narrative (posée comme 
contrainte formelle) en fictionnalise le contenu, opération inévitable dans le cas même où chaque élément du récit serait véridique, empiriquement vérifiable.

Ainsi le narrateur du récit de vie a une existence double, ambiguë, ambivalente : il est une personne en chair et en os, possédant une identité dont témoigne son état civil, entre autres traces de son indéniable existence, et un être de paroles, produit de son récit. De même, ce qui est évoqué de la réalité qui compose le cadre de l'existence du narrateur a une valeur incontestablement référentielle. Lorsque le narrateur décrit les conditions de travail, de logement de sa jeunesse, ses aventures pendant l'Occupation, le déroulement cahotant de sa vie conjugale et les peines de son enfance, il ne peut faire aucun doute qu'il reconnaît ainsi, comme le souligne avec force $\mathrm{P}$. Ricœur à propos du récit historique, sa dette envers les morts - les morts seraient-ils les différents personnages que tour à tour il dut, au cours de son existence, assumer. Ce dont il parle a bien existé ; on en peut encore saisir les traces, inscrites dans le paysage, les pierres, les façades des immeubles, sur le visage même du narrateur où passent les émotions de la réminiscence. On peut supposer que le même souci de véracité qui habite le narrateur dans la poursuite de sa vérité personnelle le contraint à ne rien omettre des faits qu'il juge importants pour l'information du destinataire de son récit : événements personnels, aventures collectives, description de contextes matériels, psychologiques, etc. Le rythme du récit dépend du nombre et de la nature des événements marquants, ceux qui instaurent un tournant dans l'existence narrée, et de la manière dont ils sont introduits et leurs effets répercutés par la narration.

6 Finalement, je reformulerai ainsi la question qui me paraît centrale pour notre propos: comment se succèdent, tels du moins qu'ils apparaissent dans les récits, les âges de la vie, selon quelle logique, en fonction de quel déterminisme externe, imposé par la force des choses, l'impact de l'histoire collective sur les vies individuelles, manifestation de la dimension référentielle du récit de vie, ou de quelle finalité narrative interne ? Y a-t-il homologie, parallélisme entre le découpage chronologique du récit de vie et les étapes de l'histoire collective ou doit-on constater une relative autonomie de la configuration narrative par rapport à la série des événements historiques majeurs retenus ou simplement évoqués? Ne peut-on, dépassant l'alternative ainsi posée, envisager un autre type de rapport entre la configuration narrative - qui renvoie à un système de valeurs plus ou moins implicite et structurant - et les événements du monde que l'individu vit, produit, subit et rêve tout ensemble, qui l'instruisent et le contraignent, le plient à leur quelquefois tragique succession?

7 Pour aborder la question de la manière la plus directe possible, il suffira de se reporter à la façon dont le narrateur articule son récit de vie en « chapitres » aisément discernables la plupart du temps, en particulier au moment de la transcription de l'enregistrement. La plupart des narrateurs respectent un ordre chronologique qu'ils identifient spontanément à l'ordre idéal du récit ; les tours et détours auxquels ils se laissent parfois aller sont rarement présentés comme libre jeu avec la norme mais comme des manquements à la règle, dus à leur inexpérience de narrateur. De ce point de vue comme de bien d'autres, il est aisé de repérer les stéréotypes à l'œuvre. On aurait tort de s'en plaindre ici, car le respect non démenti, en principe sinon toujours en pratique, de la linéarité chronologique facilite la mise en évidence des événements cruciaux, des moments qui apparaissent comme des tournants dans la vie des narrateurs.

8 À considérer les deux corpus que j'ai réunis au cours des recherches effectuées dans la Sarthe et à Paris, le résultat de ce type d'investigation ne bouleverse pas les schèmes 
établis : si, à la question de la personne interviewée qui demande par quoi elle doit commencer, on répond, sans plus de précision, "par le commencement ", "par où elle désire ", etc., c'est, conformément au modèle courant, par l'énoncé des lieu et date de naissance que le narrateur entame le plus souvent son récit. Un micro-récit de vie, un résumé de ce qui apparaît, à ce moment de l'interview, comme l'essentiel à dire, peut coiffer le récit développé ultérieurement. Comme en toute analyse de récit, la manière dont le narrateur entame son récit doit être particulièrement prise en considération : les premières phrases d'un récit de vie donnent souvent le ton, caractérisent le type de destinataire que le narrateur construit pour son récit, la problématique poursuivie, la nature de la communication qui s'instaure. Après le résumé de vie, comme marque d'une imposition de sens effectuée (ou déniée, comme cela semble être le cas lorsque ce résumé ressemble de façon trop évidente à un curriculum vitae) dans l'urgence, se développent, s'approfondissent, se singularisent, en un récit plus ou moins continu, les épisodes attendus : le récit d'enfance (le cercle familial, l'environnement, l'école, le métier...), la guerre - nous verrons quelle signification attribuer, dans les récits de vie, à cet événement dont la dimension historique est particulièrement massive -, la fondation d'une famille (rencontre du conjoint, les enfants...), la fin de la période active, la retraite, la solitude, l'attente. Cette périodisation très schématique recouvre les principales époques des récits de vie collectés. Selon les récits, chaque période est plus ou moins développée - certains développements renvoyant d'ailleurs souvent, sous leur aspect descriptif et purement informatif, au questionnement de l'intervieweur plutôt qu'au désir propre du narrateur - ce qui m'a conduit à proposer, dans la présentation de certains récits de vie, la mise en page de "hors-texte» détachés du corps du récit dont ils éclairent la compréhension sans en faire véritablement partie.

Reste à montrer que dans les récits de vie oraux - ceux, peut-être aurait-il fallu le préciser plus tôt, qui composent mon corpus -, ce ne sont pas les catégories du chercheur luimême qui s'imposent à l'interviewé et lui font adopter, outre l'ordre chronologique, le découpage de son récit en quatre parties correspondant grosso modo aux quatre âges de la vie couramment retenus: enfance, jeunesse, maturité, vieillesse. Dans la pratique de l'entretien autobiographique, on s'aperçoit assez vite que ce n'est que dans les cas où le récit de vie a des difficultés à démarrer ou se poursuivre, qu'on a recours à ce schéma classique sur lequel un consensus peut s'établir sans qu'il soit nécessaire d'en justifier le fondement. Ainsi, une fois terminé le récit d'enfance - il semblerait que le narrateur a fait le tour de la question -, si un silence s'instaure et se prolonge, il est commode de poser une question qui embraye sur la période suivante, par exemple : «À quel âge avez-vous quitté l'école?» ou «Comment avez-vous trouvé votre premier emploi? » ou encore «Comment avez-vous vécu la période de la guerre? », etc. Inversement - comme j'ai essayé de le montrer dans l'analyse concrète de quelques récits de vie en milieu rural et urbain-, un récit de vie parvenu à un degré suffisant d'élaboration - préalable au questionnement de l'intervieweur - invente ses propres enchaînements, articule divers niveaux de signification, créant ainsi une profondeur de champ qui renvoie le questionnement externe à la pauvreté d'une simple association linéaire d'idées, bref produit à la fois une structure narrative et une problématique indépendamment de la situation d'interview. Dans ces conditions, le maximum d'informations nous sera fourni, comme on pouvait s'y attendre, par le récit de vie élaboré - même court et succinct plutôt que par une série de témoignages, aussi développés et riches en informations factuelles ou autres soient-ils, portant sur les périodes successives sans que leur liaison soit établie par la personne interviewée elle-même. 
$10 \mathrm{Au}$ fond, nous pouvons seulement constater la prégnance du schéma classique des âges de la vie sur la représentation que les individus que nous avons interviewés se font de leur propre existence. Ce qui distingue les deux types de matériaux autobiographiques, c'est que dans un cas, la consécution, la suite des événements dans le temps est investie d'une signification - qui n'est pas exclusivement liée à un rapport de causalité - tandis que dans l'autre nous sommes en présence d'une succession de moments sans lien construit entre eux sinon qu'ils se rapportent tous à l'expérience d'une même personne réelle plongée dans une temporalité singulière dont le schéma classique du cycle de vie parvient à surmonter la discontinuité lorsque la singularité de l'individu ne s'affirme pas essentiellement sur le mode de la rupture, en intégrant le déroulement d'une vie particulière à une vision cosmique du temps humain. De ces témoignages ressortent, nécessairement, une "personnalité », un " caractère », ce qui est pourtant très différent de la quête identitaire à l'origine et à l'horizon de la production du récit de vie au sens fort du terme.

11 Pour résumer, je dirai que le récit de vie illustre la métaphore du cursus en la dramatisant, soulignant les moments de rupture, de crise, ceux qui engendrent les grandes décisions ou les prises de conscience, tandis que le simple entretien autobiographique se confie à l'ordre du discours dominant, induit par le questionnement $\mathrm{du}$ chercheur, pour reconstituer le cheminement sans heurt, non problématique, d'un individu porté par la logique du social. Dans ces conditions, les normes peuvent rester implicites et, finalement, questions et réponses, renvoyant, du point de vue de la personne interviewée, à un système de références partagé, à un monde commun, au système de repères incontesté, participent ensemble à la production d'un sujet d'énonciation double. Dans ce climat consensuel, que l'ordre des événements relatés et leur signification rapportée aux étapes successives d'une existence soient suggérés par le chercheur ne devrait pas être considéré pas une ingérence brutale car l'intervention du chercheur ne dynamise l'entretien qu'à la condition que ces consignes soient déjà plus ou moins intériorisées par la personne interviewée. Dans ce type de témoignage, les normes culturelles, les modèles dominants concernant les droits et les devoirs de chaque âge de la vie, les objectifs et les limitations, les déboires qui leur sont inhérents, seront fournis de façon plus directe. En effet, la manière même dont la personne interviewée choisit de témoigner de son expérience de vie inscrit le chercheur dans une relation non problématique au monde évoqué, qui n'exige pas la médiation d'un récit construisant l'identité du narrateur comme une nécessité, un préalable à toute communication. Le recours au récit complique considérablement le schéma relationnel intervieweur/ interviewé, puisque le récit comme tentative d'imposition d'un sens à un vécu multidimensionnel et polysémique, introduit la complexité propre à toute communication symbolique, surtout lorsque celle-ci s'effectue dans une société mouvante, à la hiérarchie incertaine.

12 On ne saurait cependant le déplorer, car ce n'est pas le maximum de clarté du témoignage qui constitue l'objectif du chercheur dans une démarche de sociologie qualitative. L'ambivalence, l'opacité, la difficulté d'interprétation que présente tel ou tel passage, s'insérant mal dans l'ensemble du récit, cette résistance en même temps que cette invite à l'analyse que présentent certains récits de vie - une minorité à l'intérieur de l'ensemble du matériau autobiographique collecté dans le cadre d'une enquête donnée-, sont souvent la marque de leur richesse. Le narrateur procède à un travail de réinterprétation, de mise en forme de son vécu - tentative qui ne peut lui être inspirée que par le 
sentiment diffus que l'ordre ne règne pas de façon spontanée et immédiate ou qu'un ordre doit être contesté, anéanti pour qu'un autre, plus conforme aux aspirations du sujet, se mette en place. Pour nous en tenir à la question de la périodisation, du découpage chronologique du récit de vie selon des étapes qui font avancer le sujet dans l'affirmation et la conscience de soi - si l'on reconnaît que la relation d'un tel cheminement est bien la finalité du récit de vie comme genre --, on y trouve, comme dans n'importe quel témoignage autobiographique, la reconnaissance, à un certain niveau, du déterminisme sociologique : l'insertion dans la famille, puis dans l'institution scolaire, dans le milieu professionnel, etc. constituent autant d'étapes imposées par la structure sociale, incontournables - dans nos sociétés occidentales modernes, du moins -, qu'il est donc impossible de passer complètement sous silence dans un récit qui vise à raconter l'histoire d'un individu dans le monde - conformément à la nature épique du récit de vie. Ce qu'il importe de découvrir, c'est le sens que prend, dans chaque récit de vie, chacune de ces étapes, l'importance relative qui lui est reconnue, comment le narrateur travaille le système des évidences, des normes, en singularise la signification, et par là même finit par en pervertir le fonctionnement et l'usage. On s'aperçoit alors que des récits de vie, soumis superficiellement aux contraintes du cursus ordinaire, font jouer un système parallèle à la périodisation chronologique, que je définirai comme un système de résistance achronique par cristallisations symboliques successives, grâce auquel le sujet assure la permanence de son questionnement - l'essence même de son moi problématique - face au déroulement socialement ponctué, orchestré du temps.

Ce questionnement qui supporte la conscience de soi du sujet, se dit sous forme de scènes, d'anecdotes significatives, manifestant, sur un mode quasi obsessionnel, la présence de l'enfance comme moment jamais complètement dépassé. C'est bien dans la reconstruction de son enfance que le narrateur semble vouloir trouver la clef de son rapport au monde. Mais si nous avons tous une enfance, si nous sommes tous "passés » par elle, certaines enfances paraissent marquées plus que d'autres, et marquantes au point que le sujet semble ne s'en être jamais complètement délivré - à moins que là encore nous ne puissions enregistrer qu'un effet de structure, la logique même du récit imposant que soit nouée, au plus tôt, la relation entre les différents protagonistes que la règle, implicitement acceptée, de l'unité narrative impose de tenir, jusqu'au bout, à l'horizon du récit de vie: les père et mère, accessoirement les frères et sœurs, puis le mari, figure souvent substitutive à celle du père, la communauté familiale plus ou moins large qui pose la structure relationnelle originelle, de base, entre le moi et autrui. C'est cette structure originelle que le récit figure dans une scène inaugurale où s'expriment, avec la densité de la révélation, la complexité nouvelle du monde, et la menace que la vie - le temps - fait peser sur le lien non réfléchi entre le sujet et le milieu où, en toute innocence, il baignait jusqu'alors. Cette scène correspond au nœud du récit : sans elle il semble bien que le récit de vie soit impossible à construire; je n'ai, en tout cas, collecté aucun récit de vie qui n'en offre une représentation; elle est le nœud du récit de vie compris comme développement d'une intrigue et seule une approche du témoignage autobiographique en terme d'analyse de récit permet d'en mettre en évidence la fonction structurante.

Je voudrais insister sur un point: ce n'est pas dans le caractère particulièrement dramatique d'un événement d'enfance, traumatisant, au point que l'existence même de l'adulte se résumerait à n'être qu'une sorte de quête, une recherche pour résoudre l'énigme, précocement posée, du mal et de la douleur, qu'il faut chercher l'élément 
catalyseur du récit de vie ; j'ai interviewé des femmes qui, toutes, avaient eu des enfances pour le moins difficiles, et chacune, certainement, aurait pu, dans le stock d'anecdotes qu'elle avait accumulées au cours de son enfance, trouver une histoire autour de laquelle un sens se cristallise, une scène s'impose comme lieu de convergence de multiples réseaux de signification que la suite du récit, ultérieurement, développe et clarifie. Or, une minorité seulement a su exploiter cette possibilité, la plupart s'en tenant à une description, parfois fort nourrie, du cadre (matériel, professionnel, familial, social, etc.) dans lequel les différents moments de leur existence se sont déroulés, sans recourir à une forme narrative développée à l'échelle de l'ensemble de leur vie, préférant exprimer des opinions, des jugements de valeur, de façon directe, ce qui n'exclut pas les anecdotes qui illustrent et appuient les discours.

Ce qui reste à découvrir ce sont les circonstances, extérieures à leur existence de sujets privés, qui déterminent certains individus, plus que d'autres, à organiser leurs souvenirs sous forme de récit et, de ce fait, à développer leur histoire sur le modèle romanesque de la quête identitaire, autour et à partir d'un noyau narratif qui leur fournit la première occasion d'exprimer, à travers une expression symbolisée, ce qui fonctionne ensuite comme un véritable mythe de naissance du sujet au monde. Pour aller au-delà des simples hypothèses, il faudrait disposer d'un corpus assez important pour que des variables sociodémographiques puissent être dégagées. En attendant, on peut seulement constater que ces scènes racontent la rupture, le rejet, l'expulsion qui sont le prix à consentir pour que le procès d'individuation puisse se développer. C'est pourquoi nous pouvons considérer ces scènes comme configurant un moment au statut temporel ambigu. Ce dernier fonctionne, en effet, dans le récit, comme moment du passé constamment actualisé en ce que sa réminiscence permet au sujet de reformuler, en des circonstances périlleuses dans le présent, la question, jamais totalement résolue, de son unité perdue/désirée, de sa réconciliation avec le monde. La scène inaugurale entretient le vif de la blessure et le désir d'en guérir enfin. Elle est le premier ressort de la tension qui assure au sujet la permanence de son identité dans la reconnaissance de la réalité conflictuelle du monde, qui empêche que l'illusion prenne le pas sur la vie, que le lyrisme du moi étouffe la volonté épique de surmonter les contradictions et les incertitudes.

16 Ainsi, dans le cas du récit de vie, même si, à un niveau superficiel, le narrateur respecte les étapes ordinaires d'une vie, on peut affirmer qu'au fond, son enfance ne l'a jamais lâché. On s'aperçoit aussi, à l'écoute, et plus encore à la lecture des récits de vie, qu'on ne naît pas enfant, mais qu'on entre en enfance au moment même où s'édifient les conditions de l'entrée dans l'âge adulte. Ce qui précède l'enfance est du discours rapporté ou du vécu innocent qui ne donne matière qu'à une évocation fragmentaire. L'enfance, comme enfance propre du sujet, commence dans la douleur d'une perte et d'une séparation.

Je citerai, à titre de simple illustration, un épisode, et les circonstances qui entourent son insertion dans le récit de vie, qui relate une scène d'expulsion particulièrement violente : il s'agit d'un épisode crucial de la vie de Madame B., Parisienne âgée de 68 ans au moment de l'entretien, ancienne mécanicienne en fourrure. La narratrice éprouve, de toute évidence, une grande difficulté à s'introduire dans son récit. À plusieurs reprises elle tente d'entamer le récit de sa propre vie mais c'est, à chaque fois, la biographie de sa mère qui prend le pas sur la sienne, en diffère l'introduction. Ainsi, après avoir évoqué l'installation de ses grands-parents maternels, arrivés de Russie au tout début du siècle, dans le quartier du centre de Paris qu'elle habite toujours, le métier de cordonnier du 
grand-père, sa mort - intervient alors une digression sur les conditions d'hygiène de l'époque et le décès d'un de ses copains d'enfance suite à une appendicite mal soignée -,

«Bon, alors, que je recommence... retour en arrière... donc, mes grands-parents ont eu cinq enfants et puis bon, ma mère est donc, née... »

un long passage est consacré au mariage de sa mère, très jeune, avec son père, sensiblement plus âgé qu'elle, fort laid, mais propriétaire d'une fabrique de cannes et manches de parapluie, installé dans son patrimoine. La jeune femme s'arrange pour faire une première fausse-couche, puis, enceinte à nouveau, elle tente encore de se débarrasser de cet enfant qu'elle ne désire pas :

«Elle ne voulait pas, elle s'est dit: "Non, je viens de me marier, je suis jeune." Elle voulait pas, elle voulait sortir et puis, un an après, manque de pot, je suis arrivée quand même. »

On pourrait penser que de ce constat va, tout naturellement, découler la suite du récit. Pas du tout. Madame B. continue :

«Et puis mes parents sont venus habiter la rue V..., au 5 de la rue V... Je vais vous dire comment était la rue...»

Suit une description du quartier tel qu'en lui-même la mémoire l'a fixé, « très bien tenu ", "très propre». Une deuxième possibilité d'embrayage du récit autobiographique intervient à propos du passage à l'école communale, introduite, sans préparatif, après une interruption fortuite du récit - le téléphone sonne - :

«Bon, moi, je suis allée à l'école de la rue des V..., c'était pas comme... » [Question (intempestive) : «Ça faisait maternelle aussi ?»] Réponse : «Ah, la maternelle, je suis allée rue C... - comme maintenant -, mes enfants y sont allés aussi, ça a suivi, et puis mon petit-fils; trois générations qui ont suivi dans cette école. Alors bon, après, j'ai été à l'école de la rue des V... et puis ensuite j'ai fait deux ans à l'école de la rue de M... cours complémentaires. Et puis entre-temps, mon père avait fait de mauvaises affaires dans les cannes ou manches de parapluie... ».

Notre attente est à nouveau déçue. Le récit embraye sur la malchance du père, son absence de don pour les affaires, en attendant qu'un nouvel « entre-temps » (var. : «sur ces entrefaits ») intervienne qui recentre le récit sur la figure maternelle et le cousin polonais (le territoire russe d'où la famille d'Ida était originaire ayant été rattaché à la Pologne) qui débarque et s'enrichit en créant un atelier de fourrure :

«Je sais que le cousin a gagné beaucoup d'argent. C'est arrivé en 1930, il a dû arriver en 1926. Je ne sais pas si je dois vous parler de ma naissance, comme elle s'est passée, c'était en 1918, ça s'est passé le jour de la seconde victoire de la Marne... »

Le récit de naissance, dramatique, avec, en arrière-plan, les bombardements de la Grosse Bertha, ouvre sur l'évocation de l'indifférence maternelle : celle-ci s'exprime d'abord par le refus du choix d'un prénom pour l'enfant qu'elle vient de mettre au monde. Or si le don du prénom est la première marque de reconnaissance de l'enfant comme être individualisé, cette reconnaissance, qui l'inscrit dans le groupe familial, s'effectue à partir d'une symbolique privée, intime, qui, à travers le choix du prénom, fait du nouveau-né le dépositaire des rêves, des espoirs de ses géniteurs. Or :

«Et puis mon prénom, elle me l'a donné comme ça, parce qu'elle avait entendu ce prénom-là dans la clinique, elle savait pas quel prénom donner [On notera l'élision $d u$ pronom, l'enfant n'a pas d'existence de sujet, ni même d'objet identifiable avant cette prénomination qui n'aura, de fait, jamais lieu], elle était complètement affolée et puis elle m'a donné le premier prénom qu'elle a entendu. Il y avait une voisine qui avait donné le prénom d'Ida: "Allez, ce sera Ida aussi..." Mon père a fait une comédie quand il a appris ça : "Qu'est-ce que c'est que ce prénom! - Oh", elle dit, "c'est aussi bien que n'importe lequel !" C'est tout. » 
20 prénom d'état-civil : le prénom exécré, parce que prénom de hasard, prénom qui fut le
contraire d'un don d'amour, d'une expression du libre et paradoxal choix qu'une mère fait de son enfant indépendamment de tous les déterminismes génétiques, ce prénom est tenu secret. Mais, de manière tragique, le prénom que Madame B. s'est choisi, plus tard, à l'âge adulte, devenue sujet, parce qu'il lui sert dans la mise en récit de sa naissance, se trouve du même coup entaché, souillé de tout le ressentiment, de tout le potentiel de rancœur que l'événement charrie, pour les deux principales protagonistes. Dans cet effet de récit, d'une ironie dont la cruauté n'est certainement pas perçue par le narrateur, et que seul un savoir extérieur au récit lui-même nous révèle, nous reconnaissons là le pouvoir corrosif, destructeur, de la mère que rien ne saurait contrecarrer.

Un peu plus tard nous en arrivons à l'épisode crucial qui nous intéresse : il intervient, comme le précédent, de façon brutale, comme s'il fallait violenter l'ordre du discours qui, toujours, donne la parole à la mère - ou à l'anti-figure de la mère, la voix de la raison, de la morale, qui s'exprime par la bouche d'Ida à travers des généralités qui renvoient à une communauté éthique - pour que l'individu, la personne propre, s'exprime enfin, parle en son nom, intervienne dans le dialogue qui, jusqu'à présent, n'a été rendu que par les interventions de l'Autre, principalement celles de la mère. Pour bien montrer la manière dont la narratrice s'introduit dans son récit, il me faut citer les quelques phrases qui précèdent l'épisode en question : elles concernent la façon dont on savait, dans le temps, supporter la misère, sans rien en laisser paraître, ni rien demander aux autres :

«Il y avait beaucoup plus de misère qu'à l'heure actuelle - enfin, il y en a beaucoup maintenant, c'est vrai, mais il y avait certainement autant de misère mais les gens ne prenaient pas les choses de la même façon, on volait pas, c'est tout, ça ne se faisait pas. Comme on en mendiait pas - ça vous ne mendiez pas - vous n'en aviez pas? Bien vous creviez la faim, vous vous cachiez dans un coin mais vous ne le montriez pas et puis, il ne fallait pas être sale sur soi parce qu'il ne fallait pas le montrer, et moi j'ai vécu la même chose, parce que j'ai eu une vie qui a été très dure mais j'ai jamais voulu montrer ma misère à personne. Et j'estime que si vous vous laissez aller à la misère et à mendier et tout ça, vous ne vous en sortez pas, c'est fini, vous êtes entrâné là-dedans, jamais, jamais, jamais vous ne vous en sortez. Tandis que si vous avez une certaine fierté, automatiquement vous avez envie de vous en sortir, vous avez envie de vous en tirer et il n'y a rien à faire, vous ne montrez pas votre misère, on n'a plus de respect pour vous, c'est fini, alors, vous êtes fichu, automatiquement.

Alors bon, j'en arrive à moi, à ma propre vie. Bon, ma vie, il s'est passé donc, entre ma mère et moi, il s'est passé quelque chose de très grave, c'est que moi, à l'âge de 6 ans, j'étais une gamine très vive, j'étais très en avance, je disais tout ce que je voyais, je racontais des histoires, j'étais très gaie. Et puis un jour, je devais avoir 6 ans, ma mère m'envoyait faire des courses : va chercher ceci, va chercher cela, et j'avais été rue M... - au coin de la rue des V..., il y avait une petite épicerie, là, et ma mère m'avait envoyée faire des commissions là, et il pleuvait et puis comme un fait exprès, il y avait deux jeunes gens qui étaient sous un parapluie et puis, ma foi, ils s'embrassaient et puis la fille avait mis la main, hein, bon... il s'est passé quelque chose. Moi, les gosses qui sont à la hauteur, ils voient tout ce qu'ils devraient pas voir, moi, j'arrive à la maison et puis, en riant, parce que ça m'avait fait rire [Interruption: le téléphone sonne] et puis je raconte ça en riant à ma mère, en riant aux éclats.

Ma mère, elle n'aurait pas dû faire attention, elle aurait dû me laisser raconter et puis, les enfants, ça voit les choses et puis ils ont tout de suite oublié et puis ils se disent : j'ai peut-être... c'est pas ça... Elle aurait dû dire : c'est pas ça, ou ne pas me répondre, mais alors, elle est rentrée dans une colère, elle me dit : "Je vais te sortir 
le vice de la peau." Ça n'était pas plus le vice qu'autre chose. Je ne sais pas ce qu'il lui a pris, elle m'est tombée dessus, elle m'a battue, mais battue comme j'avais jamais été battue... Je ne comprenais pas ce qui m'arrivait, ce qui me tombait et de ce jour-là, j'ai commencé à avoir une haine pour ma mère, mais une haine terrible. Et bon, ben... à partir de ce moment-là, entre elle et moi, c'était à couteaux tirés et puis alors, en plus de ça, elle-même... Alors j'étais constamment en révolte contre ma mère. Alors, plus j'étais en révolte, plus elle me tapait, et puis elle montait la tête à mon père, ce qui fait qu'à partir de cet âge-là, il ne s'est pas passé un jour de mon enfance où je n'ai pas reçu une raclée, mais pas un jour. J'avais des bleus partout, j'étais rouée de coups, si bien qu'un jour... et puis alors, le pire, c'est que je tenais tête, c'est que je tenais tête et qu'à la fin, je tenais tellement à ma mère que quand elle m'avait bien tapée, que j'avais vraiment envie de pleurer, je retenais mes sanglots et je lui disais : "Mais tu ne m'as pas fait mal." Au point qu'un jour ma mère a voulu m'étrangler et que c'est mon frère qui est venu, qui m'a arrachée de ses mains. Et puis un jour qui n'était pas comme les autres, la colère l'a prise, elle m'a prise et elle m'a descendue à la cave, je devais avoir 8 ans à cette époque-là, elle m'a enfermée à la cave et puis il y avait un petit voisin qui était à côté [...] et ce garçon, il a bien vu que ma mère m'enfermait dans la cave. Qu'est-ce qu'il a fait ? Il a ouvert la cave et puis il m'a violée et puis il m'a dit : "Si tu le dis à ta mère, elle va te battre encore et ce sera bien fait pour toi. Si tu ne dis rien, voilà.” Et il est venu m'apporter une sucette. Je suis montée en pleurant, et je n'ai jamais rien dit à ma mère et je n'ai jamais pu le lui dire jusqu'à... et elle est morte sans le savoir parce que je n'ai jamais pu le lui dire, parce que je sais que si je le lui avais dit, elle, elle m'aurait tuée, et puis même plus tard, elle m'aurait accusée de je ne sais quoi. La communication était devenue impossible et puis même avec mon père, parce qu'elle lui montait la tête ».

22 On voudra bien excuser la longueur de cette citation. La qualité et la richesse de la narration, son exceptionnelle cohérence, oblige à n'en rien soustraire - hormis un court passage qui risquerait de trahir l'anonymat des protagonistes. Faute de temps, je me contenterai de souligner les traits les plus significatifs de ce récit.

1. On notera tout d'abord sa fluidité, mise en évidence par la facilité avec laquelle Madame $B$. reprend le cours de son récit après l'interruption téléphonique, qu'elle expédie d'ailleurs avec une grande célérité. Tous les éléments s'enchaînent, il n'y a ni remords, ni détours, la narratrice maîtrise parfaitement ce qu'elle a à raconter.

24 2. Il s'agit bien de raconter les événements déterminants d'une existence, scène inaugurale ( «j'en arrive à moi, à ma propre histoire. Bon, ma vie, il s'est passé donc, entre ma mère et moi, quelque chose de très grave... »), mythe fondateur où le rapport au langage et au corps subit une métamorphose irréversible: les mots perdent leur innocence, le monde est obscurément travaillé par les forces du mal qui s'emparent d'abord des corps et cherchent ensuite à faire plier la volonté, la sexualité est vice et violence. L'apparence et l'essence se dissocient. Au lieu du rire qui libère, invite au partage, s'impose le silence de celle à qui il sera désormais interdit de raconter ses histoires, les petites histoires que trame le quotidien, histoires multiples qui tirent leur sens de se rapporter toutes à un monde partagé, commun, que les mots décrivent sans ambage parce qu'il n'a rien à cacher. Dans cette scène, l'obscurité naturelle, inhérente à la matérialité simple du recoin, de l'abri, du ventre maternel, de l'aile de la poule, perd de sa fonction protectrice : la porte cochère où l'on s'abrite de la pluie, élément transparent, eau purificatrice, devient lieu de perdition, encoignure sombre où se dissimulent les étranges pratiques, les sursauts du corps; dans la cave, où règne le noir presque absolu du tombeau, se perpétue le viol, dernière étape du martyre du corps, de sa négation : relevons l'usage absolutisé de l'expression qui renvoie habituellement à une action inscrite dans une temporalité 
courte : «j'étais rouée de coups ", être battue devient en quelque sorte l'essence du sujet, sa définition même, et sa raison d'être puisque dans l'acceptation de son état elle puise l'énergie de sa révolte: dénégation de la souffrance qui devient le recours de la victime contre la violence du maitre, résistance à la douleur à travers laquelle elle parvient à affirmer son existence; renversement dialectique de la fonction du silence : au lieu des cris, des larmes que réclame la punition maternelle, l'enfant affiche un mutisme qu'on ne peut tout à fait qualifier d'héroïque car il n'est encore que résistance passive, mais qui prépare le renversement ultérieur du rapport de force entre les deux protagonistes.

Notons au passage que ce renversement qui est l'objet même de la quête de Madame B. et qu'elle ne saurait tout à fait conquérir ni sous sa forme rasitive de l'amour (qui abolit la séparation), ni sous sa forme négative de la haine absolue (qui permet de ne rien céder de sa vérité, et ainsi de nier l'autre comme intervenant nécessairement dans toute relation communicationnelle $)^{1}$ se manifeste essentiellement par la prise de parole au sein de dialogues rapportés dans lesquels la narratrice figure en position dominante, soit qu'elle prenne, contre le désir de l'autre de garder son secret, la décision d'entamer un débat de clarification, soit qu'elle réponde, avec un sang-froid qui exprime sa maîtrise de la situation, aux questions qui lui sont posées, etc.

3. La fonction de rupture de la scène dans son ensemble par rapport au monde d'avant est fortement soulignée par la narratrice elle-même: on notera, en particulier, les expressions qui indiquent l'occurrence d'un événement inouï dans le déroulement ordinaire du temps, événement qui, au lieu de s'inscrire dans le quotidien comme confirmation d'un savoir sur le monde, se fait révélation du sens caché des choses: on entre dès lors dans la linéarité d'une histoire qui vise, peut-être, à renouer avec l'origine, objet nostalgique du désir, mais au terme d'un parcours dans le temps vécu du sujet individuel qui, lui, ne se renouvelle pas. S'il existe bien une représentation cyclique du temps rapporté au cosmos et au renouvellement des générations, elle ne résiste pas à l'épreuve de la rupture avec l'ordre traditionnel qui, libérant l'individu de la plupart des liens qui l'attachent à sa communauté naturelle (perçue, de l'intérieur, comme telle), lui fait connaître la finitude de sa propre existence. Dans le récit de Madame B., nous reconnaissons les conséquences du rejet maternel sur l'ensemble du réseau des relations primaires : dichotomie entre le groupe parental (le père, figure achevée du perdant, secondant, sans mauvaise intention, la mère) et le groupe des pairs (frère qui sauve, voisin qui viole), avec, au sein de chaque groupe, la figure d'autorité qui intime l'ordre de se taire, autorité qui n'est pas fondée sur le respect (cf. les " en plus de ça, elle-même... ", phrase inachevée qui sous-entend, comme la suite du récit le confirmera, que la mère n'a pas un comportement d'une chasteté aussi stricte que celle qu'elle exige des propos de sa fille) mais sur la crainte à cause de la violence qui en est l'apanage.

Ainsi, la rupture avec le monde des adultes n'est pas compensée par l'intégration à une communauté de remplacement: l'enfant rejeté reste seul parce que le caractère anomique de son expérience le condamne au silence, jusqu'à ce que la question du pourquoi de cette injustice dont il est victime trouve un début de réponse. Alors le récit pourra se mettre en place, à partir de la découverte d'une vérité qui concerne le rapport du sujet au désir de sa mère : et tout sombrera dans le dérisoire. Au cours d'une scène d'explication qui, pour la première fois dans le récit de Madame B., donne à la narratrice le rôle actif dans un dialogue avec sa mère - sauf le «tu ne m'as pas fait mal » enfantin, seules les paroles de la mère ou des tiers étaient rapportées, le reste du récit se faisant à la troisième personne -, la fille va soutirer à sa mère le motif de son rejet : il se résume à 
la déception qu'éprouva cette dernière à constater le manque d'attrait physique de sa fille; la mère continue cependant d'exercer sa fascination : le récit permet d'exorciser un peu de son pouvoir - une part du secret est découvert qui fonde la possibilité du récit sans toutefois poursuivre son anéantissement comme personnage central: la figure maternelle doit conserver une certaine grandeur, sinon, c'est toute l'entreprise de quête identitaire qui s'effectue par rapport à elle qui, du même coup, se trouverait diminuée. C'est pourquoi, malgré l'aveu du banal secret d'amour-propre, Madame B., la narratrice, est capable de nous faire partager une admiration, jamais démentie, pour sa mère, qui reste, à ses yeux, l'incarnation de l'énergie malfaisante et toujours victorieuse, figure glorieuse du mensonge qui s'oppose à la faiblesse honnête et impuissante du père.

4. Cette scène marque, sur un mode conforme à la position dominée du sujet dans le rapport à la mère, le début du procès d'individuation: remarquons simplement, inscrite dans le contexte de la scène, l'ambivalence de la formule: «Mais tu ne m'as pas fait mal. ». S'adressant à sa mère, Ida lui dit deux choses :

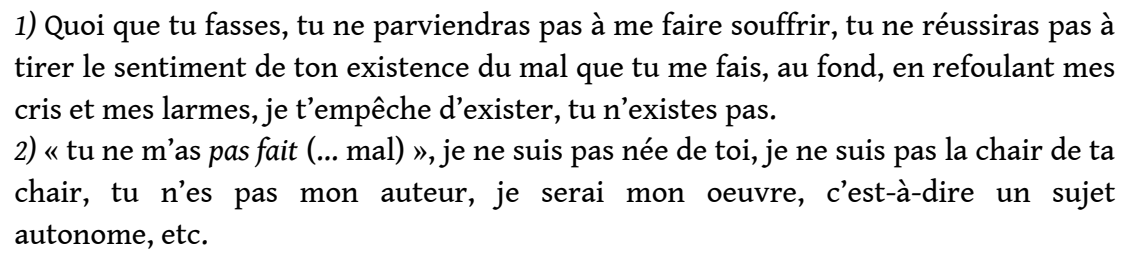

5. Cette scène, dont j'ai dit que s'y révélait l'enjeu du récit de vie, jette le sujet dans le temps linéaire d'une histoire qui débute par un rejet et aspire à un dénouement incertain. L'irruption de cette violence redoublée qui sera désormais le sel de son existence, la narratrice l'évoque en jouant, avec beaucoup d'art, sur l'opposition entre un événement qui n'entretient avec ce qui précède aucun lien logique perceptible, du point de vue de l'enfant qui subit sans comprendre ( « je ne sais pas ce qu'il lui a pris... Je ne comprenais pas ce qui m'arrivait, ce qui me tombait...») et les conséquences de cette première brutalité sans motif apparent, conséquences qui, elles, se soumettent à la dialectique du maître et de l'esclave, s'inscrivent dans une chronologie parfaitement restituée d'escalade de la violence, de montée aux extrêmes (« de ce jour-là j'ai commencé... et à partir de ce moment-là... alors j'étais constamment en révolte contre ma mère, alors plus j'étais en révolte, plus elle me tapait... ce qui fait qu'à partir de cet âge-là... si bien qu'un jour... et puis alors, le pire... je tenais tellement tête que... au point qu'un jour... »), jusqu'à la chute dans les enfers, la cave, où la révolte se mue, victoire maternelle, en larmes et en silence. À partir de ce «jour qui n'était pas comme les autres », le recours à la chronologie n'a plus de sens : le silence n'a pas d'histoire. Pourtant, après le viol, le secret a changé de camp, c'est Ida qui devient désormais celle qui sait, qui connait, mieux que sa mère, le véritable motif de leur haine qui a, certainement, à voir avec le père, l'origine juive du père, que son nom dissimule (la grand-mère paternelle est juive polonaise, le grand-père lorrain, catholique) mais que dénoncent son physique, son accent, les enfants d'un premier mariage ${ }^{2}$, ses habitudes alimentaires, etc.

Avec l'épisode de la guerre, Madame B. prend véritablement le contrôle de son récit : amère revanche sur la mère puisqu'elle la doit à la menace qui pèse sur cette dernière à cause de ses origines juives. Alors, pour la première fois, la ressemblance d'Ida avec son père, l'espèce de connivence qui rapproche les deux victimes d'un même acharnement mauvais, joue en sa faveur, car si c'est le père qui a transmis le patronyme français, insignifiant, grâce auquel la famille parviendra à se sauver, c'est Ida qui sera l'agent actif du salut collectif. La période de la guerre occupe dans le récit de vie de Madame B. une 
place considérable : ses prises de paroles se multiplient, c'est elle qui prend les décisions, qui convainc sa mère de fuir en zone libre, jusqu'à Marseille, la sauvant du même coup de la déportation, cache des membres de sa famille et des amis juifs, prend contact avec la Résistance, affronte en toute conscience les risques et s'en sort toujours par son sangfroid. Nous suivons, épisode après épisode, les aventures de la guerre et les démêlés d'Ida avec sa mère, vie privée, histoire collective mêlées, Ida tenant son rôle dans les deux contextes, servant d'intermédiaire à ceux que l'histoire oblige à se tenir cachés, condamnés, à leur tour, à l'obscurité des greniers et des caves. On découvre une dimension pathétique à ce dévouement hérö̈que, complaisamment dévoilé, aux membres d'une communauté à laquelle elle n'avoue son appartenance qu'au moment où elle aborde l'épisode de la guerre, l'ayant dissimulée lors même qu'elle évoquait l'origine de ses grands-parents maternels dont elle n'indiquait que la nationalité russe sans préciser qu'ils étaient également juifs. Nous sommes bien dans une structure d'épreuve, l'événement historique, majeur, guerre, persécution raciale, déportation, risque de mort, toute l'horreur tragique d'une expérience collective démesurée, que Madame B. a bien vécue, apparaît comme l'occasion fournie au Moi de s'insurger contre l'autorité maternelle, de prendre le pouvoir dans un récit qui multiplie les indices de succès dans les épreuves successives, succès par ruse, revanche de l'intelligence sur l'occupant, sur la mère, accès, enfin, à l'âge adulte, celui de l'autonomie de la parole.

Les quelques remarques qui précèdent sembleront peut-être empreintes d'ironie ou de cynisme à l'égard du récit d'Ida. Il n'en est rien. D'une part, cette «utilisation» de la guerre par le sujet qui se met en scène sur fond d'héroïsme attesté par l'historiographie officielle, est une pratique courante dans les récits de vie de tous ceux qui ont vécu cette époque : il est même curieux de constater avec quelle facilité les personnes les moins disposées à mettre leur vie en récit, produisent, sur la guerre, des morceaux narratifs qui leur offrent l'occasion de se singulariser, d'introduire les discours rapportés, indices de fictionnalisation du récit, d'une prise de distance par rapport à l'univers évoqué, etc. D'autre part, il me semble que la structure même du récit impose, outre la scène inaugurale que j'ai longuement analysée dans une de ses réalisations exemplaires mais qu'on trouve, j'oserais dire nécessairement, à l'origine de tous les récits de vie, et qui marque le passage de l'état fusionnel à l'enfance proprement dite, comme état de conscience sans pouvoir, la présence d'un épisode où la maturité du personnagenarrateur éclate: l'état de guerre me paraît particulièrement propice à ce type d'affirmation puisqu'il correspond à un affaiblissement du pouvoir des pères (ou mères) - tout particulièrement dans une situation d'occupation où l'autorité légitime a révélé son impuissance et sa couardise -, à la remise du sort commun entre les mains des plus jeunes (ce sont eux qui se battent et meurent), bref à un désordre généralisé de la société qui profite à ceux qu'on a trop longtemps fait taire. Dans les récits concernant la guerre, les femmes, par exemple, parlent, beaucoup plus qu'à l'ordinaire, en leur nom, même celles de la campagne habituées à s'exprimer à la troisième personne, en usant du « on » anonyme et collectif. En fait, on devrait dire que, dans la perspective de production des récits de vie, renvoyant au procès d'individuation dans les sociétés occidentales modernes, si la guerre n'avait pas existé, il faudrait l'inventer... ou un événement possédant des traits structuraux équivalents, susceptible d'occuper une fonction similaire dans l'histoire du sujet.

32 À vrai dire, une fois ces deux épisodes mis en place, scène inaugurale et scène héroïque, les éléments du récit de vie se distribuent sans beaucoup de surprise, confirmant, 
développant, enrichissant la structure originelle où s'articulent les deux événements. Pour conclure, je serais tentée de dire - mais je ne pense pas être la première - que le récit de vie est un récit de mort, parce qu'il est construit à partir d'un lieu de savoir, de sagesse, d'immobilité intérieure, de sérénité conquise contre le mouvement même de la vie, avec ses incertitudes et ses risques, et parce qu'il n'a de sens qu'à poursuivre la mort de ceux qui vous ont précédés, qui ont imposé au monde l'ordre de leur discours, l'ont modelé à l'image de leurs propres angoisses, de leurs propres fantasmes. Inversement, il reste bien récit de vie car le narrateur est, au fond, soutenu dans son entreprise par le sentiment confus qu'on ne se débarrasse jamais de son enfance. Comme dans le roman classique, la linéarité du récit, la chronologie apparaît bien comme une conquête du sujet, la forme d'un dépassement possible de la structure régressive et de la répétition, ouverture proposée et niée dès la scène inaugurale, miniaturisation du récit comme champ de tension, réalisée en apparence dans la scène héroïque, mais réfutée par l'existence même du récit. Car, pourquoi raconter quand on a tout compris? Donnons une fois encore la parole à Ida :

« Alors, quand j'étais enfant, j'attendais d'être adulte pour avoir des droits et je me suis aperçue qu'étant adulte j'avais encore ma mère et que je n'avais pas de droits quand même. »

\section{NOTES}

1. Ainsi elle n'osera jamais raconter le viol à sa mère, crainte de n'être pas crue, d'être accusée, jugée, ce qui constitue l'aveu pathétique de sa dépendance à l'égard de cette mère énigmatique, admirée et détestée à la fois, dépendance jusqu'à la mort, sa propre mort puisque la disparition de sa mère rend irrémédiable son impuissance à dire, à faire (à mettre) définitivement le point sur (à) leur histoire.

2. Il s'est enfui, enfant, de chez son père, remarié après la mort de sa mère, pour rejoindre, en Pologne, le village de cette dernière, où il est resté jusqu'à l'âge d'homme. Il rentre finalement en France, laissant sa jeune femme, juive polonaise qui, pendant son absence prolongée à cause de la guerre de 14 qui éclate à ce moment-là, meurt d'une épidémie de choléra, laissant deux enfants qui, en 1920, débarquent à leur tour à Paris, chez leur père qui, entre-temps, a fondé une nouvelle famille, étant resté sans nouvelles de sa famille polonaise... 